\title{
Effects of sustained hypoxia on sternohyoid and diaphragm muscle during development
}

\author{
Jayne C. Carberry ${ }^{1}$, Clodagh McMorrow ${ }^{1}$, Aidan Bradford ${ }^{2}$, James F.X. Jones ${ }^{1}$ \\ and Ken D. O'Halloran ${ }^{3}$
}

Affiliations: 'School of Medicine and Medical Science, University College Dublin, Dublin, ${ }^{2}$ Dept of Physiology and Medical Physics, Royal College of Surgeons in Ireland, Dublin, and ${ }^{3}$ Dept of Physiology, School of Medicine, University College Cork, Cork, Ireland.

Correspondence: J.C. Carberry, Neuroscience Research Australia, PO Box 1165, Sydney, NSW 2031, Australia. E-mail: j.carberryaneura.edu.au

ABSTRACT Sustained hypoxia is a dominant feature of respiratory disease. Despite the clinical significance, the effects of sustained hypoxia on the form and function of respiratory muscle during development are relatively underexplored.

Wistar rats were exposed to 1 week of sustained hypoxia (ambient pressure $450 \mathrm{mmHg}$ ) or normoxia at various time points during development. Sternohyoid and diaphragm muscle contractile and endurance properties were assessed in vitro. Muscle succinate dehydrogenase and myosin heavy chain composition were determined. The role of reactive oxygen species in hypoxia-induced muscle remodelling was assessed.

Sustained hypoxia increased sternohyoid muscle force and fatigue in early but not late development, effects that persisted after return to normoxia. Hypoxia-induced sternohyoid muscle fatigue was not attributable to fibre type transitions or to a decrease in oxidative capacity. Chronic supplementation with the superoxide scavenger tempol did not prevent hypoxia-induced sternohyoid muscle fatigue, suggesting that mechanisms unrelated to oxidative stress underpin hypoxia-induced maladaptation in sternohyoid muscle. Sustained hypoxia had no effect on diaphragm muscle fatigue.

We conclude that there are critical windows during development for hypoxia-induced airway dilator muscle maladaptation. Sustained hypoxia-induced impairment of upper airway muscle endurance may persist into later life. Upper airway muscle dysfunction could have deleterious consequences for the control of pharyngeal airway calibre in vivo.

There are critical windows during development for hypoxia-induced airway dilator muscle maladaptation http://ow.ly/t7iVj

\footnotetext{
This article has supplementary material available from www.erj.ersjournals.com

Received: Sept 042012 | Accepted after revision: May 182013 | First published online: June 132013

Support statement: This study was partly funded by the Health Research Board (Ireland) (grant number RP/2008/159). J.C. Carberry was in receipt of a UCD School of Medicine and Medical Science PhD training scholarship.

Conflict of interest: Disclosures can be found alongside the online version of this article at www.erj.ersjournals.com 


\section{Introduction}

The effector organs of the respiratory control system, the striated muscles of breathing, play a critical role in oxygen and acid-base homeostasis. Respiratory muscle remodelling may have adaptive or maladaptive consequences for respiratory control. Sustained hypoxia is commonly encountered in healthy individuals at high altitude and in patients with various respiratory diseases (e.g. chronic obstructive pulmonary disease (COPD)).

Skeletal muscle has the capacity to adapt to sustained hypoxia by way of modulation of skeletal muscle vasculature [1, 2], muscle enzyme activities [3-6], muscle fibre size and distribution [7-10] and contractile performance [11-13]. Moreover, sustained hypoxia has been shown to elicit functional plasticity in respiratory muscles $[9,10]$ in a manner that differs from the phenotypic changes occurring in limb muscles $[10,14,15]$.

Hypoxia is common in premature babies and in infants with congenital heart disease or bronchopulmonary dysplasia [16, 17]. Sustained hypoxic exposure during development elicits plasticity in respiratory control $[18,19]$ and was shown to decrease diaphragm muscle specific tension [20]. Moreover, diaphragm muscles of infants having sudden infant death syndrome have reportedly fewer type I fatigue-resistant fibres than healthy infants [21]. However, in general, there is a great paucity of information concerning the effects of sustained hypoxia on respiratory muscle during development, despite the obvious clinical relevance.

We, therefore, sought to determine the effects of exposure to sustained hypoxia at different stages of early development on rat diaphragm and pharyngeal-dilator (sternohyoid) muscle contractile and endurance properties, oxidative capacity, myosin heavy-chain (MHC) fibre distribution and cross-sectional area (CSA). In addition, we sought to examine the putative role of reactive oxygen species (ROS) in sustained hypoxia-induced muscle plasticity. Hypoxia has been shown to increase free-radical species in muscle [22, 23]. Redox signalling can influence transcriptional activators leading to altered muscle phenotype [24]. Furthermore, muscle-derived ROS and nitric oxide target contractile proteins, ryanodine receptor calcium channels, sarco/endoplasmic reticulum calcium-ATPase and sodium-potassium pumps [9, 25-27], thus altering muscle contractile and endurance properties. The superoxide scavenger tempol recovered chronic intermittent hypoxia-induced upper airway muscle impairment in a rat model of sleep apnoea [28, 29]. This suggests a role for superoxide or downstream oxidants in hypoxia-induced muscle dysfunction. Therefore, given the potential clinical application, we tested the hypothesis that antioxidant treatment would prevent sustained hypoxia-induced aberrant functional remodelling in neonatal rat respiratory muscles. Part of this study was published in short form following presentation at the XIth Oxford Conference (Nara, Japan, 2009) [30].

\section{Methods}

A detailed description of the methods is provided in the online supplementary material.

\section{Animals}

Experiments were performed on Wistar rats of mixed sex. For sustained hypoxia studies, litters (with respective dams) were placed in a hypobaric chamber at an ambient pressure of $450 \mathrm{mmHg}$ for 7 days starting at postnatal day (P)1 ( $=7)$, P6 $(n=8)$ or P11 $(n=8)$; muscle function studies were conducted at $\mathrm{P} 19$. In separate litters, hypoxic exposure began in weaned animals starting at P21 or P31 and experiments were performed at P29 $(n=8)$ and P39 $(n=7)$, respectively. Age-matched controls (P19n=9, P29 n=11 and P39 $n=8)$ were maintained under normobaric conditions $(\sim 760 \mathrm{mmHg})$ in parallel. One additional litter was exposed to sustained hypoxia (starting at P11) and received daily oral administration of a superoxide scavenger, tempol (100 mg. $\mathrm{kg}^{-1}, \mathrm{n}=7$ ), starting 3 days prior to hypoxic exposure and continuing until P19. The $\mathrm{n}$ values represent the total number of animals used for each group. For some protocols, we did not obtain data from all animals due to technical difficulties and for those datasets the adjusted $\mathrm{n}$ value is reported in the figure legend.

\section{Effects of sustained hypoxia on respiratory muscle force and fatigue}

The sternohyoid and diaphragm muscles from normoxic and sustained hypoxia rats were excised and contractile and endurance properties were determined in vitro. Muscles were snap frozen in isopentane cooled by liquid nitrogen. The samples were stored at $-80^{\circ} \mathrm{C}$ for later use.

\section{Effects of sustained hypoxia on respiratory muscle MHC fibre type and oxidative enzyme activity} MHC immunocytochemistry

MHC fibre types were determined by indirect immunofluoresence with monoclonal antibodies on serial transverse sections of muscle from normoxic and sustained hypoxia groups. MHC isoforms were detected 
using a triple-labelling technique employing a cocktail of antibodies as previously described [9]. This enabled the detection of MHC I, IIA and IIB on the same tissue section. Pure MHC IIx fibres were identified on separate sections using an indirect approach [9].

\section{Succinate dehydrogenase}

Serial $10-\mu \mathrm{m}$ transverse sections of normoxic and sustained hypoxia respiratory muscles were cut at $-22^{\circ} \mathrm{C}$ on a cryostat. The activity of the mitochondrial enzyme succinate dehydrogenase (SDH) was determined as an index of the oxidative capacity of the muscles. Muscle samples from all of the experimental groups were processed together under identical conditions. Control (blank) reactions were performed with the omission of the primary substrate.

Data analysis

Specific force was calculated in $\mathrm{N} \cdot \mathrm{cm}^{-2}$ of muscle CSA. Nonlinear regression (curve-fit) analysis was employed (GraphPad Prism; GraphPad, La Jolla, CA, USA) for force-frequency relationship, allowing us to determine values for stimulus frequency producing 50\% of peak force (EF50). For the fatigue trials, muscle forces were measured at time zero (initial force) and at 1-min intervals, and a fatigue index (i.e. ratio of force at $5 \mathrm{~min}$ of fatigue to initial force) was determined. Scion Image software (Scion Corporation, Frederick, MD, USA) was used to determine optical density as a measure of SDH activity. We quantified muscle MHC fibre types by calculating their relative contribution to muscle CSA (i.e. areal density) using Cell A software (Olympus Life Science Microscopes, Munich, Germany) using four square test frames $(200 \times 200 \mu \mathrm{m}$ each $)$ per muscle section. The test frames were randomly placed over muscle sections. All data are expressed as mean \pm SEM.

\section{Statistical analysis}

Statistical comparisons were made between normoxic and sustained hypoxia groups using one-way ANOVA (body mass, contractile kinetics, peak tetanic force and fatigue index) using a Newman-Keuls post hoc test (comparing all groups) and Dunnett's post hoc test (comparing all groups to control) where appropriate. A two-tailed unpaired t-test was used to compare data sets in P29 and P39 animals (normoxia versus sustained hypoxia). $\mathrm{p}<0.05$ was the criterion for significance in all tests.

\section{Results}

Effects of sustained hypoxia on body mass, haematocrit and cardiac mass

Values are shown in the online supplementary table E1. Exposure to sustained hypoxia in early life (preweaning groups) had no significant effect on body mass (table E1). Sustained hypoxia caused a significant decrease in body mass in the P29 and P39 groups. Sustained hypoxia increased haematocrit and right ventricle mass in all groups (table E1).

\section{Effects of sustained hypoxia on respiratory muscle force and fatigue \\ Sternohyoid}

The effects of sustained hypoxia on sternohyoid muscle twitch force and contractile kinetics are shown in the online supplementary table E2. Sustained hypoxia increased sternohyoid force-frequency relationship (fig. E2) and peak force (fig. 1a) if exposed to hypoxia during the 3 weeks of life. Sustained hypoxia had no effect on peak force when sustained hypoxia exposure began in late development (P39) (fig. 1c). Sustained hypoxia did not affect sternohyoid muscle EF50 in any age group (data not shown). Sustained hypoxia significantly increased sternohyoid muscle fatigue when exposure began pre-weaning (fig. 2a) but not postweaning (figs $2 b$ and $c$ ).

\section{Diaphragm}

The effects of sustained hypoxia on diaphragm-muscle twitch force and contractile kinetics are shown in the online supplementary table E3. Sustained hypoxia significantly increased diaphragm peak isometric force in rats exposed to hypoxia beginning at P1 (fig. 3a). Sustained hypoxia had no effect on peak force in all other groups (fig. 3). Sustained hypoxia had no effect on diaphragm EF50 values (data not shown). Sustained hypoxia had no significant effect on diaphragm fatigue index (fig. 4).

\section{Effects of sustained hypoxia on respiratory muscle MHC fibre type and oxidative enzyme activity Sternohyoid}

Sustained hypoxia had no significant effect on sternohyoid muscle SDH activity at any age during development (data not shown). Representative immunofluorescent images are shown in figure 5. Areal density values and fibre CSAs are shown in table 1. Sustained hypoxia in early life (pre-weaning groups) had no effect on areal density values of MHC type I, IIA and IIB isoforms (table 1). No pure MHC IIx fibres 
were detected in P19 sternohyoid. Sustained hypoxia exposure was associated with significant fibre hypertrophy for some exposure periods during early development (table 1).

\section{Diaphragm}

Sustained hypoxia had no significant effect on diaphragm muscle SDH activity (data not shown). Representative immunofluorescent images are shown in figure 5. Areal density values and fibre CSAs are shown in table 2. Sustained hypoxia in early life (pre-weaning groups) had no major effect on areal density values and CSAs of MHC fibre types (table 2).

Effects of antioxidant treatment on sustained hypoxia-induced increase in sternohyoid muscle force and fatigue

Chronic administration of tempol (100 $\mathrm{mg} \cdot \mathrm{kg}^{-1}$ by mouth) had no effect on sustained hypoxia-induced increase in sternohyoid muscle peak force and fatigue (online supplementary fig. E1).

\section{Discussion}

The major findings of this study are: 1) sustained hypoxia increases sternohyoid (pharyngeal dilator) muscle force-frequency and fatigue, effects that persist during neonatal development; 2) sustained hypoxia-induced respiratory muscle plasticity is age-dependent and differentially expressed in airway dilator and thoracic pump muscles; 3) sustained hypoxia-induced fatigue in sternohyoid muscle is not related to changes in SDH activity or MHC isoform composition; and 4) chronic administration of the superoxide scavenger tempol does not prevent sustained hypoxia-induced sternohyoid muscle fatigue.

\section{Effects of sustained hypoxia on respiratory muscle force and fatigue}

There is a general paucity of information concerning the effects of sustained hypoxia on respiratory muscle, which is quite surprising, given the clinical significance. Respiratory muscle remodelling following sustained hypoxia has been reported in adult rats and differential effects of sustained hypoxia on airway dilator and thoracic pump muscles have been noted $[9,12]$. Of interest, diaphragm muscle is either resistant to [10-12] or even shows improved endurance [9] following sustained hypoxia. A recent study by GAMBOA and ANDRADE [10] highlights the potential importance of a downregulation in uncoupling protein-3 in hypoxic adaptation in respiratory muscle. This adaptation may be unique to the diaphragm, as sustained hypoxia causes limb muscle fatigue $[10,14,15]$ and fatigue is also reported for the adult sternohyoid muscle in one [12] but not another [9] study. The present study extends these observations showing that 1 week of sustained hypoxia is sufficient to cause sternohyoid muscle dysfunction in neonatal rats when the exposure occurs early in life. It is also apparent that there are differential effects of sustained hypoxia within respiratory muscles (sternohyoid versus diaphragm), which is consistent with previous studies on limb muscles $[11,13,14]$. Whether the functional effects of sustained hypoxia are dependent upon the structural makeup and metabolic profiles of various muscles remains to be determined. Slow-twitch and fast-twitch fibres differ in ATP utilisation, oxidative capacity and blood flow distribution, thus it is plausible that decreased oxygen availability might have diverse consequences for muscle physiology. Interestingly, Howlett and Hogan [31] showed that muscle composed of glycolytic fibres is more susceptible to decreased oxygen supply in vivo compared with muscle composed of oxidative fibres.

In this light, differences in structural phenotype may explain the observation of age-dependent plasticity following sustained hypoxia. The structural makeup of skeletal muscle undergoes developmental changes towards the adult phenotype from birth. Respiratory muscles in rat undergo significant changes in fibre type profile during the first month of life $[32,33]$. KASS and BAZZY [20] found that the impairment of diaphragm muscle force following sustained hypoxia decreased with age. In addition, we predicted age-dependent muscle plasticity. In our study, sustained hypoxia exposure in early developing animals (first month of life) resulted in alterations in sternohyoid muscle function and these effects persisted for several days upon return to normoxia. Conversely, when animals were exposed to the same hypoxic stimulus at an older age, no functional effects were observed. Thus, there would appear to be critical periods during development when the sternohyoid muscle is most vulnerable to hypoxia-induced increased fatigue. Moreover, it appears that sustained hypoxia-induced functional effects persist for at least as long as the initial sustained hypoxia exposure. Whether the functional changes reported here for the sternohyoid, namely increased force and fatigue, represent adaptive or maladaptive remodelling is less clear, but these changes may have consequences for upper airway control in later life. Our results suggest the intriguing possibility that early life hypoxia may predispose to upper airway instability in later life, including increased propensity for obstructive apnoea [34, 35]. 
Effects of sustained hypoxia on respiratory muscle MHC fibre type and oxidative enzyme activity The mechanism for the sustained hypoxia-induced decrease in sternohyoid endurance is unknown. Changes in skeletal muscle endurance are often associated with altered oxidative enzyme activities [36-38]. In humans, reductions in oxidative capacity following hypoxia have been reported $[4,7,39$, 40]. However, sustained hypoxia-induced changes in sternohyoid muscle endurance were not attributable to changes in SDH activity. This contrasts with other animal studies, where sustained hypoxia decreased oxidative enzyme activity [5, 6, 41]. However, in these studies, hypoxic exposures ranged from 2 to 10 weeks. We acknowledge that only one oxidative enzyme was assessed in our study and it is possible that the activity of others (e.g. citrate synthase or cytochrome c oxidase) was decreased. Other studies have reported reductions in oxidative enzyme activity in limb muscles concomitant with increases in glycolytic enzyme activity $[4,41]$ following sustained hypoxia. In our study, glycolytic enzyme activity was not measured. Recently, our laboratory has shown that sternohyoid muscle fatigue during development correlates with age-related increases in glycerol phosphate dehydrogenase (GPDH) activity [42]. Increased GPDH activity while SDH activity remains unchanged alters the oxidative/glycolytic ratio. Thus, it appears that a decrease in the ratio of oxidative to glycolytic enzymes results in a more fatiguable muscle during development [42]. It is possible that glycolytic enzyme activity is enhanced during sustained hypoxia. Further investigation of respiratory muscle glycolytic enzyme activity following sustained hypoxia may yield clues as to the mechanisms underpinning hypoxia-induced sternohyoid muscle dysfunction.

We postulated that changes in MHC isoform expression were responsible for the changes in muscle contractility. A slow-to-fast fibre transition would be consistent with the increases in sternohyoid isometric force and fatigue that we observed. However, sustained hypoxia did not affect MHC areal density in the sternohyoid, thus a slow-to-fast phenotypic transition does not underlie the hypoxia-induced alterations in muscle contractility and endurance. Reports in the literature of the effects of sustained hypoxia on skeletal muscle phenotype are conflicting. Human studies have reported increased proportions of slow fibres in high-altitude natives compared to sea-level dwellers [43, 44]. However, in simulated altitude studies, no change in fibre type profile was found [39]. In limb muscles of COPD patients, there is evidence of a reduced complement of slow fibres with shifts towards higher proportions of fast fibres [45-48]. In the diaphragm of COPD patients, it is thought that the reverse happens, i.e. increased numbers of slow fibres and a decreased proportion of IIa fibres $[49,50]$, but while COPD is characterised by chronic hypoxia, the disease is complex and many other factors contribute to respiratory muscle remodelling.

Conflicting reports concerning hypoxia-related fibre type transitions extend to animal models of sustained hypoxia. Some studies report no significant change in fibre distribution following sustained hypoxia $[9,51,52]$. Mortola and NASO [53] showed that 9 months of hypoxia ( $\sim 14 \%$ oxygen), but not 60 days, induced slow-to-fast fibre type transitions in rodent limb and diaphragm muscle. Conversely, BIGARD et al. [54] showed that 4 weeks of sustained hypoxia was sufficient to induce slow-to-fast fibre transitions in the soleus of the developing rat, although the hypoxic exposure was more severe $(\sim 10 \%$ oxygen $)$. Similar reports of a slow-to-fast fibre transition have been reported in adult rat limb muscles following 4 and 5 weeks of sustained hypoxia at $10 \%$ and $\sim 15 \%$ oxygen, respectively $[14,55]$. SHIOTA et al. [11] reported a shift from fast to slow phenotype in the extensor digitorum longus (EDL) muscle, with no reported changes in the soleus muscle. A 10-week exposure to sustained hypoxia in developing rats
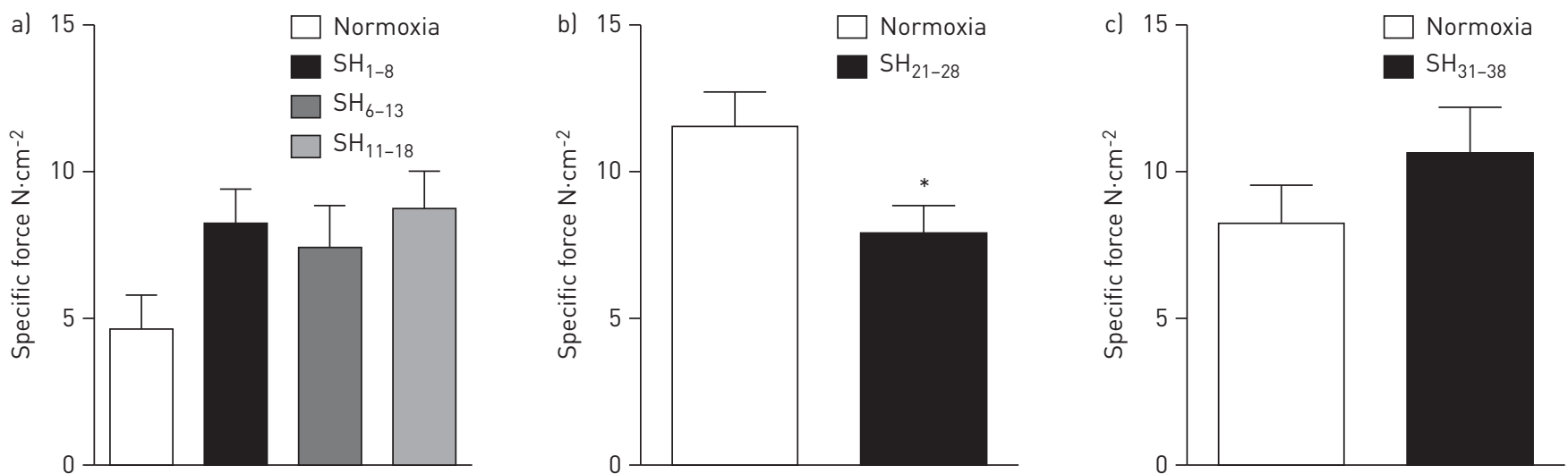

FIGURE 1 Mean \pm SEM values for sternohyoid peak isometric force in rats exposed to normoxia or sustained hypoxia (SH) for 7 days starting at post-natal day (P) 1, P6, P11, P21 or P31. Muscle studies were performed at a) P19, b) P29 and c) P39. Statistical analysis was performed using a) one-way ANOVA (p=0.14) and unpaired $\mathrm{t}$-test for $\mathrm{b})(\mathrm{p}=0.05)$ and $\mathrm{c})(\mathrm{p}=0.27)$. For force-frequency relationship (P19) see the online supplementary figure E2. $\mathrm{n}=6-8$ for all groups. *: $\mathrm{p}=0.05$. 

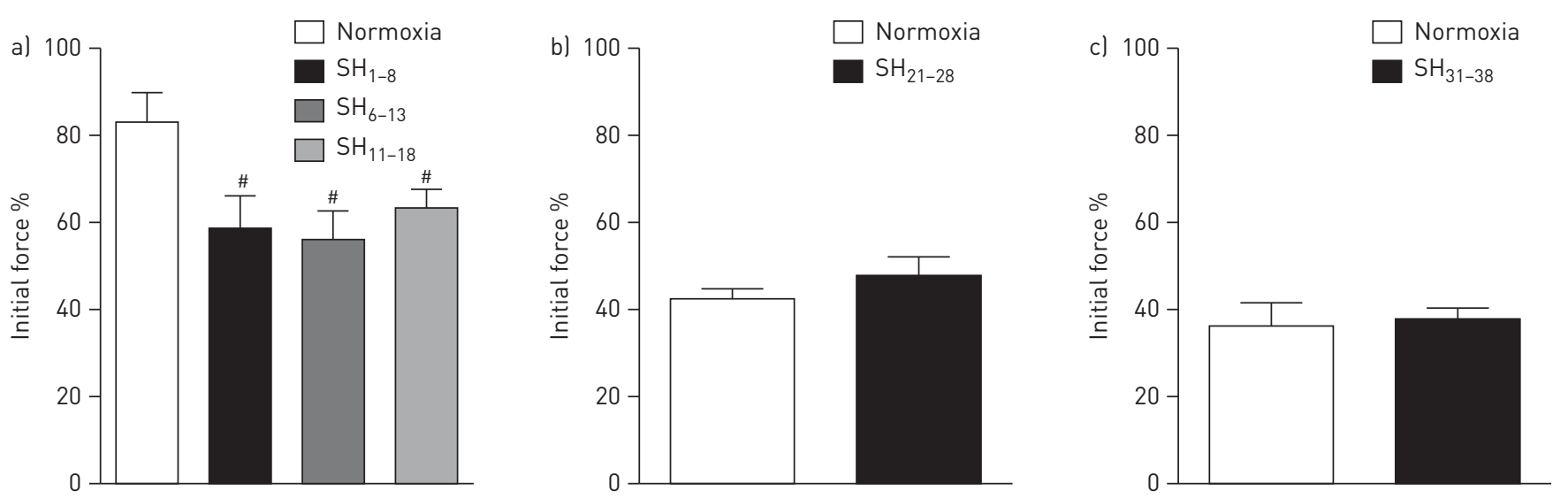

FIGURE 2 Mean \pm SEM values for sternohyoid fatigue index in rats exposed to normoxia or sustained hypoxia (SH) for 7 days starting at post-natal day (P)1, P6, P11, P21 or P31. Muscle studies were performed at a) P19, b) P29 and c) P39. Statistical analysis was performed using a) one-way ANOVA (p=0.02) with Dunnett's post hoc test $(\mathrm{p}<0.05), \mathrm{b})$ unpaired $\mathrm{t}$-test $(\mathrm{p}=0.26)$, and $\mathrm{c})$ Mann-Whitney $\mathrm{U}$-test $(\mathrm{p}=0.28) . \mathrm{n}=6-8$ for all groups. * : significant difference from normoxia.

(aged 1 month) caused a relative increase in the proportion of IIa fibres and decrease in the slow fibre distribution of the soleus muscle, whereas the EDL increased in IIa but decreased in IIb fibre proportions [13]. Thus, it appears that hypoxia-induced fibre-type transitions depend on the structural makeup of the muscle $[11,13]$. This supports the notion that fast-twitch muscles are more susceptible to fatigue than slow-twitch muscles during sustained hypoxia. It has also been shown that moderate systemic hypoxia can affect muscle fibre types in different regions within a muscle [56]. Thus, results may depend on the method of analysis (whole muscle versus regional areas). Potential findings may be lost if regional data are grouped [56]. In our older animals, the sternohyoid muscle was cut into longitudinal strips for structural and functional analysis. This may have influenced our findings if regional effects are a factor. In addition, it should be noted that structural and functional remodelling is probably dependent on the intensity and duration of hypoxic exposure, making comparisons between different studies difficult to interpret.

Effects of antioxidant treatment on sustained hypoxia-induced increase in sternohyoid muscle force and fatigue

Tempol is a stable membrane permeable nitroxide compound that acts as a superoxide dismutase (SOD) mimetic and a superoxide scavenger [57]. Superoxide scavengers have been shown to protect diaphragm maximum tetanic force in hypoxia in vitro [58]. Furthermore, tempol was effective in the protection of superoxide-induced impairment in tetanic force and maximum calcium-activated force due to heat stress [59]
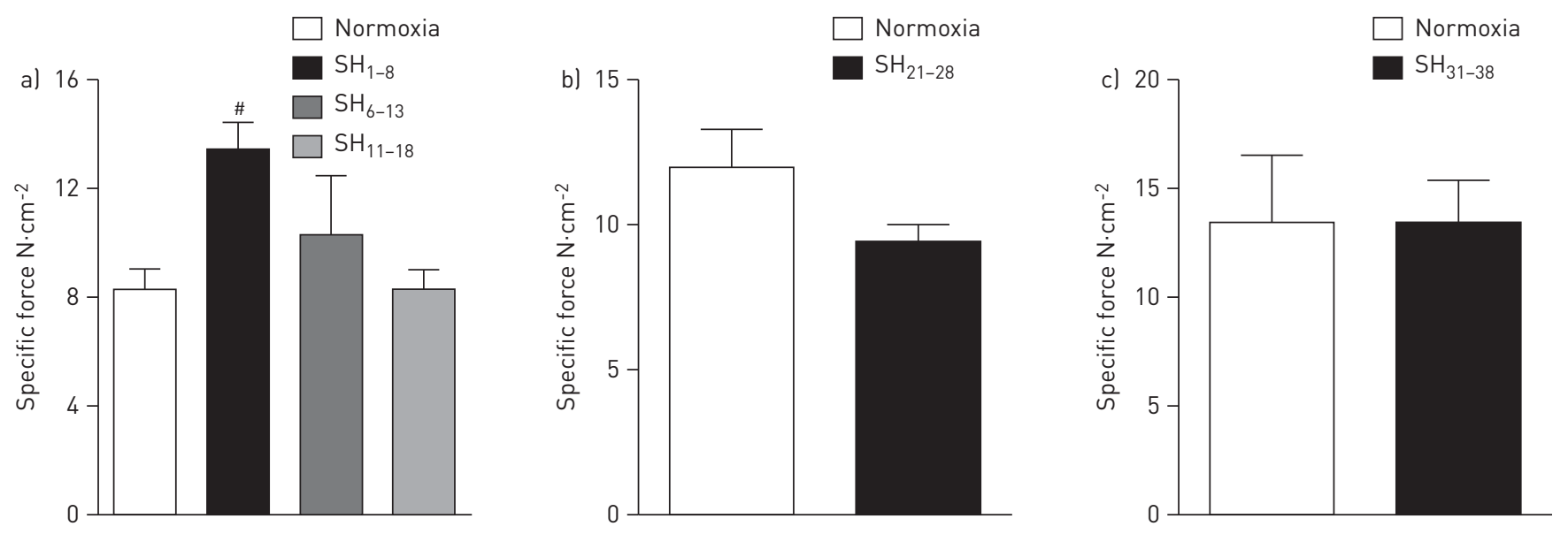

FIGURE 3 Mean \pm SEM values for diaphragm peak isometric force in rats exposed to normoxia or sustained hypoxia (SH) for 7 days starting at post-natal day (P) 1, P6, P11, P21 or P31. Muscle studies were performed at a) P19, b) P29 and c) P39. Statistical analysis was performed using a) one-way ANOVA ( $\mathrm{p}=0.02$ ) with Dunnett's post hoc test $(\mathrm{p}<0.05)$ and unpaired $\mathrm{t}$-test for $\mathrm{b})(\mathrm{p}=0.09)$ and $\mathrm{c})(\mathrm{p}=0.99) . \mathrm{n}=5-8$ for all groups. ${ }^{*}$ : indicates significant difference from normoxia. 

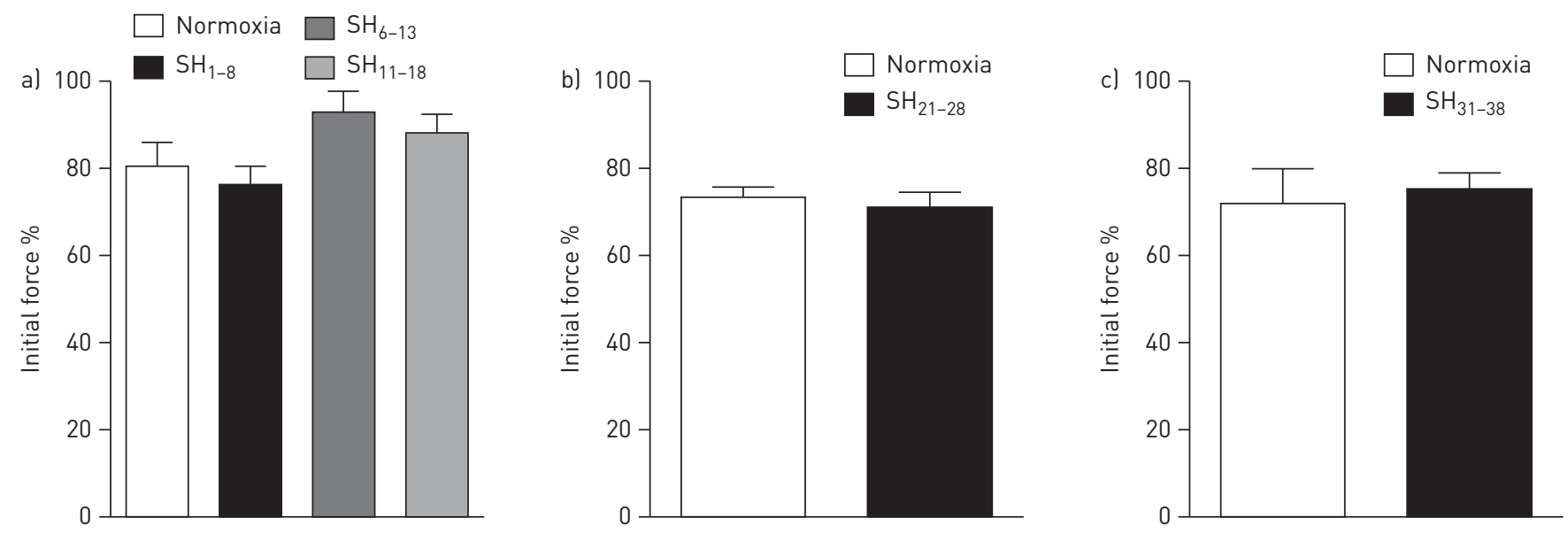

FIGURE 4 Mean \pm SEM values for diaphragm fatigue index in rats exposed to normoxia or sustained hypoxia (SH) for 7 days starting at post-natal day (P)1, P6, P11, P21 or P31. Muscle studies were performed at a) P19, b) P29 and c) P39. Statistical analysis was performed using a) one-way ANOVA (p=0.08), and unpaired $\mathrm{t}$-test for $\mathrm{b})(\mathrm{p}=0.58)$ and $\mathrm{c})(\mathrm{p}=0.76) . \mathrm{n}=5-8$ for all groups.

and hypoxia [28]. In adult rats, tempol administered in vitro improved upper airway muscle tension, an effect that persisted during repeated muscle activation [28]. Moreover, tempol-incubated adult sternohyoid showed improved force and performance in the early phase of fatigue during hypoxia [28]. Recent studies in our laboratory have shown that daily administration of tempol prevents chronic intermittent hypoxia-induced upper airway muscle dysfunction [29]. We speculated that oxidative stress was also implicated in sustained hypoxia-induced sternohyoid muscle fatigue. However, daily administration of tempol to neonatal pups did not prevent sustained hypoxia-induced effects in the sternohyoid muscle. Our finding suggests that oxidative stress does not contribute to hypoxia-induced functional remodelling in sternohyoid muscle during development. In isolated perfused rat diaphragm, significant increases in superoxide during fatigue were
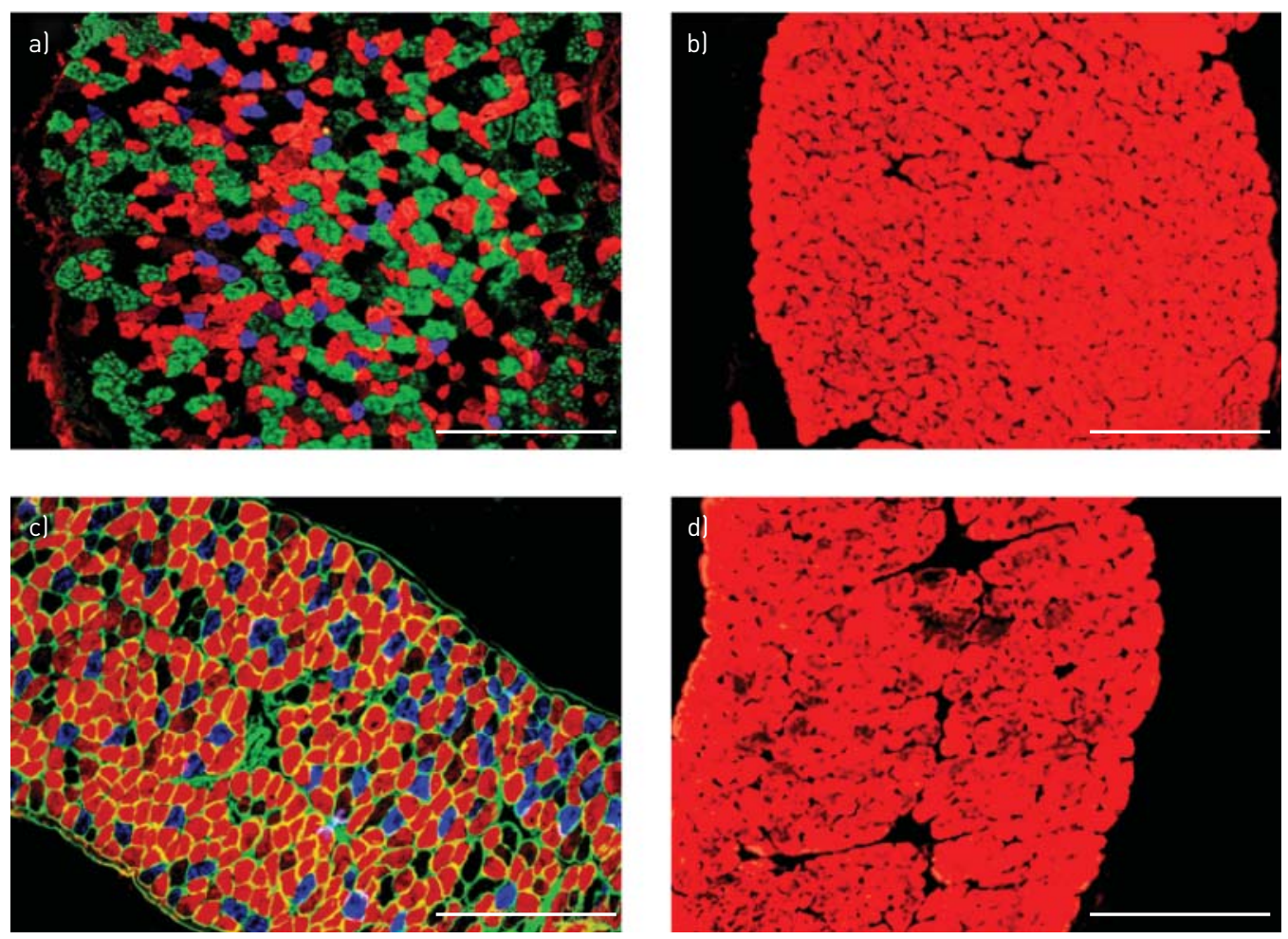

FIGURE 5 Immunofluorescent images of and post-natal day (P)19 a) sternohyoid and c) diaphragm following incubation with antibodies against myosin heavy chain (MHC) I (blue), IIa (red) and IIb (green). P19 b) sternohyoid and d) diaphragm muscle following incubation with a pan-MHC antibody tagging all fibres (red) except MHC IIx. The basement membrane protein laminin is labelled (green) in c). Scale bars $=200 \mu \mathrm{m}$. 
TABLE 1 Sternohyoid muscle fibre areal density and cross-sectional area (CSA) in normoxic and sustained hypoxia rats at post-natal day (P)19

\begin{tabular}{lcccc} 
& Normoxia & SH $_{\mathbf{1 - 8}}$ & $\mathbf{S H}_{\mathbf{6 - 1 3}}$ & SH $_{\mathbf{1 1 - 1 8}}$ \\
\hline Areal density \% & & & & \\
I & $7 \pm 1$ & $10 \pm 2$ & $7 \pm 1$ & $9 \pm 1$ \\
Ila & $27 \pm 2$ & $32 \pm 3$ & $31 \pm 3$ & $30 \pm 2$ \\
IIx & & & $40 \pm 4$ \\
IIb & $41 \pm 2$ & $36 \pm 2$ & $30 \pm 4$ & $477 \pm 24^{*}$ \\
I m $^{2}$ & $379 \pm 14$ & $495 \pm 20^{*}$ & $374 \pm 25$ & $414 \pm 39$ \\
Ila & $334 \pm 14$ & $408 \pm 31$ & $339 \pm 21$ & $0 \pm 0$ \\
IIx & $0 \pm 0$ & $0 \pm 0$ & $0 \pm 0$ & $1017 \pm 84^{*}$ \\
Ilb & $746 \pm 51$ & $790 \pm 41$ & $651 \pm 32$ & \\
\hline
\end{tabular}

Data are presented as mean \pm SEM. There were no pure IIx fibres in the P19 sternohyoid muscle. SH: sustained hypoxia. Statistical analysis was performed using Kruskal-Wallis nonparametric tests with Dunn's multiple comparison test or one-way ANOVA followed by Dunnett's post hoc test where appropriate. $\mathrm{n}=5-9$ for all groups. *: significant difference from normoxia, $p<0.05$.

determined by measuring the level of cytochrome $\mathrm{c}$ reduction but SOD did not ameliorate diaphragm fatigue [60]. As such, SOD-mimetics may not always be the best approach for protection of skeletal muscle function during oxidative stress (hypoxia). Alternatively, other free radicals such as nitric oxide may have a more important regulatory role in hypoxia-induced muscle plasticity. Indeed, recent findings from our laboratory have implicated nitric oxide in diaphragm muscle adaptation to sustained hypoxic stress [9]. Additionally, we have shown that nitric oxide synthase (NOS) inhibition in vitro has a positive inotropic effect on the sternohyoid at P19 (unpublished data), consistent with reports that nitric oxide has an inhibitory action in skeletal muscle contractility [61]. Sustained hypoxia may alter sternohyoid muscle NOS expression and activity, and such changes may underpin the functional changes reported in this study.

\section{Limits and strengths of the study}

Our data are derived from isolated muscle function studies. This preparation offers many advantages, allowing a study of the effects of sustained hypoxia on respiratory myocyte function under controlled conditions (stimulus, oxygen, temperature, etc.), such that changes in muscle contractile and endurance properties can be attributed to intrinsic changes at the level of the muscle itself. However, this approach also has significant limitations. It is difficult to translate the observation of sternohyoid muscle fatigue to the in vivo setting, where, for example, there may be sufficient or otherwise neural compensation for increased muscle fatigue. As such, we do not know if the control of airway calibre is adversely affected in sustained

TABLE 2. Diaphragm muscle fibre areal density and cross-sectional area (CSA) in normoxic and sustained hypoxia rats

\begin{tabular}{lcccc} 
& Normoxia & SH $_{1-8}$ & SH $_{6-13}$ & SH $_{11-18}$ \\
\hline Areal density \% & & & & \\
I & $21 \pm 2$ & $22 \pm 3$ & $18 \pm 5$ & $20 \pm 3$ \\
Ila & $53 \pm 4$ & $60 \pm 3$ & $60 \pm 1$ & $40 \pm 2^{*}$ \\
IIx & $0 \pm 0$ & $0 \pm 0$ & $0 \pm 0$ & $6 \pm 3^{*}$ \\
IIb & $0 \pm 0$ & $1 \pm 1$ & $1 \pm 1$ & $3 \pm 1^{*}$ \\
CSA m $^{2}$ & & & & $712 \pm 34$ \\
I & $663 \pm 53$ & $621 \pm 41$ & $600 \pm 45$ & $688 \pm 19$ \\
Ila & $671 \pm 49$ & $1351(1 / 6)$ & $730(1 / 6)$ & $1089 \pm 102(4 / 7)$ \\
Ilx & & & $1184 \pm 180(3 / 7)$ \\
\hline
\end{tabular}

Data are presented as mean \pm SEM, mean $(n / N)$ or mean \pm SEM $(n / N)$. There were no pure IIx and Ilb fibres in the post-natal day 19 normoxic diaphragm muscle. SH: sustained hypoxia. Statistical analysis was performed using Kruskal-Wallis non-parametric tests with Dunn's multiple comparison test or one-way ANOVA followed by Dunnett's post hoc test where appropriate. $n=6-9$ for all groups. *: significant difference from normoxia, $\mathrm{p}<0.05$. 
hypoxia-treated rats. Moreover, our data are derived from an animal model and readers should exercise caution in extrapolating the findings to human infants, although we argue that the findings may have relevance to neonatal hypoxia. However, it is not clear if sustained hypoxia during early life in humans is associated with airway dilator muscle dysfunction and studies addressing this gap in our knowledge are necessary. The group sizes in our study are relatively small and it is possible that some comparisons (particularly in the context of respiratory muscle structure) are underpowered for statistical purposes, perhaps contributing to the apparent lack of effect for some parameters.

\section{Summary and conclusion}

In summary, we observed sustained hypoxia-induced functional effects in neonatal but not adolescent sternohyoid muscle. Conversely, the diaphragm muscle was resistant to hypoxia-induced functional change. Our findings are consistent with the view that there are critical "windows" during development wherein hypoxia-induced maladaptation may occur with potentially long-lasting effects. Functional alterations in the sternohyoid muscle were not attributable to fibre type transitions or decreased oxidative capacity. Antioxidant treatment did not prevent sustained hypoxia-induced sternohyoid muscle fatigue, suggesting that oxidative stress is not the underlying mechanism driving hypoxic maladaptation in developing sternohyoid muscle. Respiratory muscle impairment is implicated in a range of respiratory disorders and dysfunction can ultimately lead to respiratory failure. Adjunct therapies aimed at improving airway dilator muscle performance during hypoxia may prove beneficial in the treatment of respiratory disorders. Thus, the molecular mechanism of hypoxia-induced muscle dysfunction warrants further investigation.

\section{References}

1 Deveci D, Marshall JM, Egginton S. Chronic hypoxia induces prolonged angiogenesis in skeletal muscles of rat. Exp Physiol 2002; 87: 287-291.

2 Snyder GK, Wilcox EE, Burnham EW. Effects of hypoxia on muscle capillarity in rats. Respir Physiol 1985; 62: $135-140$.

3 Wüst RC, Jaspers RT, van Heijst AF, et al. Region-specific adaptations in determinants of rat skeletal muscle oxygenation to chronic hypoxia. Am J Physiol Heart Circ Physiol 2009; 297: H364-H374.

4 Howald H, Pette D, Simoneau JA, et al. Effect of chronic hypoxia on muscle enzyme activities. Int J Sports Med 1990; 11: Suppl. 1, S10-S14.

5 Takahashi H, Kikuchi K, Nakayama H. Effect of chronic hypoxia on oxidative enzyme activity in rat skeletal muscle. Ann Physiol Anthropol 1993; 12: 363-369.

6 Abdelmalki A, Fimbel S, Mayet-Sornay MH, et al. Aerobic capacity and skeletal muscle properties of normoxic and hypoxic rats in response to training. Pflugers Arch 1996; 431: 671-679.

7 Hoppeler H, Kleinert E, Schlegel C, et al. Morphological adaptations of human skeletal muscle to chronic hypoxia. Int J Sports Med 1990; 11: Suppl. 1, S3-S9.

8 MacDougall JD, Green HJ, Sutton JR, et al. Operation Everest II: structural adaptations in skeletal muscle in response to extreme simulated altitude. Acta Physiol Scand 1991; 142: 421-427.

9 McMorrow C, Fredsted A, Carberry J, et al. Chronic hypoxia increases rat diaphragm muscle endurance and sodium-potassium ATPase pump content. Eur Respir J 2011; 37: 1474-1481.

10 Gamboa JL, Andrade FH. Muscle endurance and mitochondrial function after chronic normobaric hypoxia: contrast of respiratory and limb muscles. Pflugers Arch 2012; 463: 327-338.

11 Shiota S, Okada T, Naitoh H, et al. Hypoxia and hypercapnia affect contractile and histological properties of rat diaphragm and hind limb muscles. Pathophysiology 2004; 11: 23-30.

12 El-Khoury R, O'Halloran KD, Bradford A. Effects of chronic hypobaric hypoxia on contractile properties of rat sternohyoid and diaphragm muscles. Clin Exp Pharmacol Physiol 2003; 30: 551-554.

13 Itoh K, Moritani T, Ishida K, et al. Hypoxia-induced fibre type transformation in rat hindlimb muscles. Histochemical and electro-mechanical changes. Eur J Appl Physiol Occup Physiol 1990; 60: 331-336.

14 Faucher M, Guillot C, Marqueste T, et al. Matched adaptations of electrophysiological, physiological, and histological properties of skeletal muscles in response to chronic hypoxia. Pflugers Arch 2005; 450: 45-52.

15 El-Khoury R, Bradford A, O'Halloran KD. Chronic hypobaric hypoxia increases isolated rat fast-twitch and slowtwitch limb muscle force and fatigue. Physiol Res 2012; 61: 195-201.

16 Chess PR, D’Angio CT, Pryhuber GS, et al. Pathogenesis of bronchopulmonary dysplasia. Semin Perinatol 2006; 30: $171-178$.

17 Aisenberg RB, Rosenthal A, Nadas AS, et al. Developmental delay in infants with congenital heart disease. Correlation with hypoxemia and congestive heart failure. Pediatr Cardiol 1982; 3: 133-137.

18 Bavis RW, Olson EB, Vidruk EH, et al. Developmental plasticity of the hypoxic ventilatory response in rats induced by neonatal hypoxia. J Physiol 2004; 557: 645-660.

19 Carroll JL. Developmental plasticity in respiratory control. J Appl Physiol 2003; 94: 375-389.

20 Kass LJ, Bazzy AR. Chronic hypoxia modulates diaphragm function in the developing rat. J Appl Physiol 2001; 90: 2325-2329.

21 Lamont P, Chow C, Hilton J, et al. Differences in diaphragm fiber types in SIDS infants. J Neuropathol Exp Neurol 1995; 54: 32-37.

22 Clanton TL. Hypoxia-induced reactive oxygen species formation in skeletal muscle. J Appl Physiol 2007; 102: 2379-2388.

23 Zuo L, Clanton TL. Reactive oxygen species formation in the transition to hypoxia in skeletal muscle. Am J Physiol Cell Physiol 2005; 289: C207-C216.

24 Schiaffino S, Sandri M, Murgia M. Activity-dependent signaling pathways controlling muscle diversity and plasticity. Physiology (Bethesda) 2007; 22: 269-278. 

myotubes. J Appl Physiol 2001; 91: 2117-2124.

al. Protein modification during biological aging: selective tyrosine nitration of the SERCA2a isoform of the sarcoplasmic reticulum $\mathrm{Ca}^{2+}$-ATPase in skeletal muscle. Biochem J 1999; 340: 657-669.

McKenna MJ, Medved I, Goodman CA, et al. $\mathrm{N}$-acetylcysteine attenuates the decline in muscle $\mathrm{Na}^{+}, \mathrm{K}^{+}$-pump activity and delays fatigue during prolonged exercise in humans. J Physiol 2006; 576: 279-288.

28 Skelly JR, Bradford A, Jones JF, et al. Superoxide scavengers improve rat pharyngeal dilator muscle performance. Am J Respir Cell Mol Biol 2010; 42: 725-731.

29 Skelly JR, Edge D, Shortt CM, et al. Tempol ameliorates pharyngeal dilator muscle dysfunction in a rodent model of chronic intermittent hypoxia. Am J Respir Cell Mol Biol 2012; 46: 139-148.

30 Carberry J, Bradford A, O’Halloran KD. Antioxidant treatment does not prevent chronic hypoxia-induced respiratory muscle impairment in developing rats. Adv Exp Med Biol 2010; 669: 263-266.

31 Howlett RA, Hogan MC. Effect of hypoxia on fatigue development in rat muscle composed of different fibre types. Exp Physiol 2007; 92: 887-894.

32 Punkt K, Naupert A, Asmussen G. Differentiation of rat skeletal muscle fibres during development and ageing. Acta Histochem 2004; 106: 145-154.

33 Johnson BD, Wilson LE, Zhan WZ, et al. Contractile properties of the developing diaphragm correlate with myosin heavy chain phenotype. J Appl Physiol 1994; 77: 481-487.

34 Sharma PB, Baroody F, Gozal D, et al. Obstructive sleep apnea in the formerly preterm infant: an overlooked diagnosis. Front Neurol 2011; 2: 73.

35 Hibbs AM, Johnson NL, Rosen CL, et al. Prenatal and neonatal risk factors for sleep disordered breathing in schoolaged children born preterm. J Pediatr 2008; 153: 176-182.

36 Puente-Maestu L, Tena T, Trascasa C, et al. Training improves muscle oxidative capacity and oxygenation recovery kinetics in patients with chronic obstructive pulmonary disease. Eur J Appl Physiol 2003; 88: 580-587.

37 Henriksson J. Training induced adaptation of skeletal muscle and metabolism during submaximal exercise. J Physiol 1977; 270: 661-675.

38 Lewis MI, Zhan WZ, Sieck GC. Adaptations of the diaphragm in emphysema. J Appl Physiol 1992; 72: 934-943.

39 Green HJ, Sutton JR, Cymerman A, et al. Operation Everest II: adaptations in human skeletal muscle. J Appl Physiol 1989; 66: 2454-2461.

40 Cerretelli P. Muscle energetics and ultrastructure in chronic hypoxia. Respiration 1992; 59: Suppl. 2, $24-29$.

41 De Palma S, Ripamonti M, Vigano A, et al. Metabolic modulation induced by chronic hypoxia in rats using a comparative proteomic analysis of skeletal muscle tissue. J Proteome Res 2007; 6: 1974-1984.

42 O'Connell RA. Intermittent hypoxia/reoxygenation increases respiratory muscle fatigability in neonatal rats: no role for reactive oxygen species. Proc Physiol Soc 2010; 19: C102.

43 Rosser BW, Hochachka PW. Metabolic capacity of muscle fibers from high-altitude natives. Eur J Appl Physiol Occup Physiol 1993; 67: 513-517.

44 Kayser B, Hoppeler H, Desplanches D, et al. Muscle ultrastructure and biochemistry of lowland Tibetans. J Appl Physiol 1996; 81: 419-425.

45 Maltais F, Sullivan MJ, LeBlanc P, et al. Altered expression of myosin heavy chain in the vastus lateralis muscle in patients with COPD. Eur Respir J 1999; 13: 850-854.

46 Jakobsson P, Jorfeldt L, Brundin A. Skeletal muscle metabolites and fibre types in patients with advanced chronic obstructive pulmonary disease (COPD), with and without chronic respiratory failure. Eur Respir J 1990; 3: 192-196.

47 Hildebrand IL, Sylvén C, Esbjörnsson M, et al. Does chronic hypoxaemia induce transformations of fibre types? Acta Physiol Scand 1991; 141: 435-439.

48 Whittom F, Jobin J, Simard PM, et al. Histochemical and morphological characteristics of the vastus lateralis muscle in patients with chronic obstructive pulmonary disease. Med Sci Sports Exerc 1998; 30: 1467-1474.

49 Levine S, Kaiser L, Leferovich J, et al. Cellular adaptations in the diaphragm in chronic obstructive pulmonary disease. N Engl J Med 1997; 337: 1799-1806.

50 Mercadier JJ, Schwartz K, Schiaffino S, et al. Myosin heavy chain gene expression changes in the diaphragm of patients with chronic lung hyperinflation. Am J Physiol 1998; 274: L527-L534.

51 Takahashi H, Kikuchi K, Nakayama H. Effect of chronic hypoxia on skeletal muscle fiber type in adult male rats. Ann Physiol Anthropol 1992; 11: 625-630.

52 Sillau AH, Banchero N. Effect of hypoxia on the capillarity of guinea pig skeletal muscle. Proc Soc Exp Biol Med 1979; 160: 368-373.

53 Mortola JP, Naso L. Electrophoretic analysis of contractile proteins of the diaphragm in chronically hypoxic rats. Am J Physiol 1995; 269: L371-L376.

54 Bigard AX, Sanchez H, Birot O, et al. Myosin heavy chain composition of skeletal muscles in young rats growing under hypobaric hypoxia conditions. J Appl Physiol 2000; 88: 479-486.

55 Roels B, Reggiani C, Reboul C, et al. Paradoxical effects of endurance training and chronic hypoxia on myofibrillar ATPase activity. Am J Physiol Regul Integr Comp Physiol 2008; 294: R1911-R1918.

56 Deveci D, Marshall JM, Egginton S. Relationship between capillary angiogenesis, fiber type, and fiber size in chronic systemic hypoxia. Am J Physiol Heart Circ Physiol 2001; 281: H241-H252.

57 Krishna MC, Russo A, Mitchell JB, et al. Do nitroxide antioxidants act as scavengers of $\mathrm{O}_{2}{ }^{-}$or as SOD mimics? J Biol Chem 1996; 271: 26026-26031.

58 Wright VP, Klawitter PF, Iscru DF, et al. Superoxide scavengers augment contractile but not energetic responses to hypoxia in rat diaphragm. J Appl Physiol 2005; 98: 1753-1760.

59 Edwards JN, Macdonald WA, van der Poel $\mathrm{C}$, et al. $\mathrm{O}_{2} \cdot^{-}$production at $37^{\circ} \mathrm{C}$ plays a critical role in depressing tetanic force of isolated rat and mouse skeletal muscle. Am J Physiol Cell Physiol 2007; 293: C650-C660.

60 Kolbeck RC, She ZW, Callahan LA, et al. Increased superoxide production during fatigue in the perfused rat diaphragm. Am J Respir Crit Care Med 1997; 156: 140-145.

61 Kobzik L, Reid MB, Bredt DS, et al. Nitric oxide in skeletal muscle. Nature 1994; 372: 546-548. 